\title{
Hemisacrectomy with preservation of the contralateral sacral nerve roots and sacroiliac joint for pelvic neurofibrosarcoma in a 7-year-old child: case report with 2-year follow-up
}

\author{
Ali H. Palejwala, BS, ${ }^{1}$ Jared S. Fridley, MD, ${ }^{1}$ Krystal Garcia, BS, ${ }^{1}$ Sanjeev A. Vasudevan, MD, ${ }^{2}$ \\ David Khechoyan, MD, ${ }^{3}$ Surya Rednam, MD, ${ }^{4}$ Chester J. Koh, MD, ${ }^{5}$ and Andrew Jea, MD ${ }^{1,6}$
}

\begin{abstract}
${ }^{1}$ Neuro-Spine Program, Division of Pediatric Neurosurgery; ${ }^{2}$ Division of Pediatric Surgery; ${ }^{3}$ Division of Pediatric Plastic Surgery, Texas Children's Hospital; “Division of Pediatric Hematology/Oncology, Texas Children's Cancer Center; ${ }^{5}$ Division of Pediatric Urology, Texas Children's Hospital, Houston, Texas; and ${ }^{6}$ Section of Pediatric Neurosurgery, Riley Hospital for Children, Indiana University School of Medicine Department of Neurosurgery, Indianapolis, Indiana
\end{abstract}

\begin{abstract}
Neurofibrosarcoma is rare in the pediatric age group. A malignant tumor of the sacrum presents significant challenges, especially if the goals are to resect with wide and clean surgical margins and to achieve acceptable functional outcomes. The authors report a case of this rare tumor affecting the sacrum and sacral nerve roots of a 7-year-old girl and review the role of total hemisacrectomy sparing the contralateral sacral nerve roots and lumbopelvic reconstruction in the treatment of this disease. This patient is, to the best of the authors' knowledge, the youngest to be treated in this manner.
\end{abstract}

http://thejns.org/doi/abs/10.3171/2016.7.PEDS16203

KEY WORDS hemisacrectomy; neurofibrosarcoma; pediatric spine; sacral tumor; sacrectomy; oncology

$\mathrm{N}$ EUROFIBROSARCOMA is a nonrhabdomyosarcoma soft-tissue sarcoma arising in peripheral nerve sheath tissue. They are rare tumors of neuroectodermal origin, composing between $4 \%$ and $10 \%$ of all soft-tissue sarcomas in the pediatric population..$^{16}$ Resecting neurofibrosarcomas in children can be difficult. These tumors often appear in proximity to major nerves and vessels. A neurofibrosarcoma arising from sacral nerve roots and proximal sciatic nerve in the pelvic cavity and extending through the sciatic notch presents with similar challenges.

Most malignancies originating from the sacrum or pelvis are not sensitive to chemotherapy or radiotherapy, and resection is the mainstay therapy. ${ }^{5,6,8,15}$ Sacral malignancies treated with gross-total resection and total sacrectomy sacrifice bilateral sacral nerve roots. Postoperative complications such as urinary and fecal incontinence are therefore inevitable. We previously reported our experience with total sacrectomy in a 15-year-old girl with sacral myofibroblastic sarcoma. ${ }^{13}$

In a limited number of cases, however, unilateral sacral nerve roots can be preserved during tumor resection. ${ }^{5}$
Preservation of these sacral nerve roots is expected theoretically to improve neurological function. While technically demanding, ensuring a tumor surgical margin may be possible via hemisacrectomy through a sagittal plane in the sacrum without sacrificing the contralateral sacral nerve roots or sacroiliac joint. ${ }^{15}$ We performed this procedure on a 7-year-old girl with a new diagnosis of neurofibrosarcoma originating from the right sciatic nerve and involving multiple sacral nerve roots. Herein, we report the tumor resection technique, lumbopelvic reconstruction strategy, and postoperative functional and oncological outcomes at 25 months after surgery. To the best of our knowledge, our patient is the youngest to have undergone a total hemisacrectomy.

\section{Case Report}

History and Physical Examination

This 7-year-old girl had a history of progressively worsening right leg pain over the course of 3 months. Eventually, she was so debilitated from pain that she was unable to walk. At the same time, she developed difficulty with 

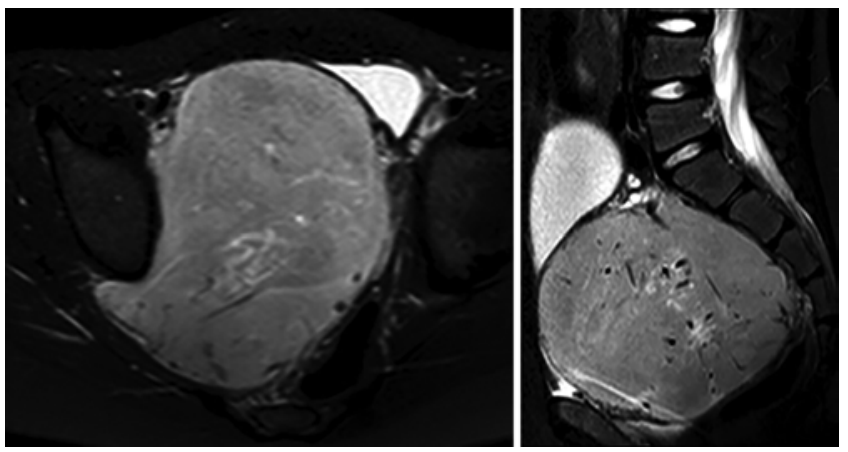

FIG. 1. Axial (left) and sagittal (right) T2-weighted MR images showing a large hyperintense pelvic mass with notable vascular flow voids. The bladder is compressed and shifted right to left. The epicenter of the large tumor is off to the right of midline with extension through the sciatic notch. The sacral nerve roots and proximal sciatic nerve are not visible.

bladder emptying and voiding. A workup revealed a large pelvic mass (Fig. 1). The patient was taken to the operating room at an outside institution for an excisional biopsy via a transabdominal approach. Pathological analysis revealed schwannoma.

Subsequently, the patient was transferred to Texas Children's Hospital for further care. Our pediatric surgery team performed a partial resection of the tumor via a transabdominal approach based on the prior pathology. However, pathological analysis at our institution revealed a high-grade malignant peripheral nerve sheath tumor (MPNST). A staging workup showed no disease in other remote sites. At this point, without any reasonable options for chemotherapy or radiotherapy, we planned a 2-stage anterior-posterior surgery to remove the residual gross tumor with an attempt at achieving surgical margins.

\section{Operation}

A 2-stage total hemisacrectomy was planned in a multidisciplinary manner, as previously described..$^{5,15}$ The first stage included teams from pediatric surgery, urology, and plastic surgery. A redo midline laparotomy, mobilization of the visceral and neural structures, and reexcision and reestablishment of tumor margins (extirpation of right psoas muscle in contact with the tumor), as confirmed by sampling and frozen pathology, were performed. The ovaries were also mobilized and fixed outside of the pelvic cavity in preparation for postoperative radiation therapy. The posterior right bladder wall and distal right ureter in contact with the tumor were resected; margins were negative for tumor. The bladder wall was closed with an endto-end reimplantation of the right ureter. Bilateral ureteral stents were placed. An ileostomy was performed, and a left rectus abdominus myocutaneous flap with oblique skin paddle fed by the inferior epigastric vessels was mobilized, wrapped in a bowel bag, and then placed in the pelvis.

The second stage of the surgery was performed on the following day. A midline skin incision from the spinous process of L-3 to the tip of the coccyx was extended into the posterior right thigh to provide a trans- and infragluteal exposure of the sciatic nerve from the sciatic notch. Tumor was inseparable from the sciatic nerve as it pro-

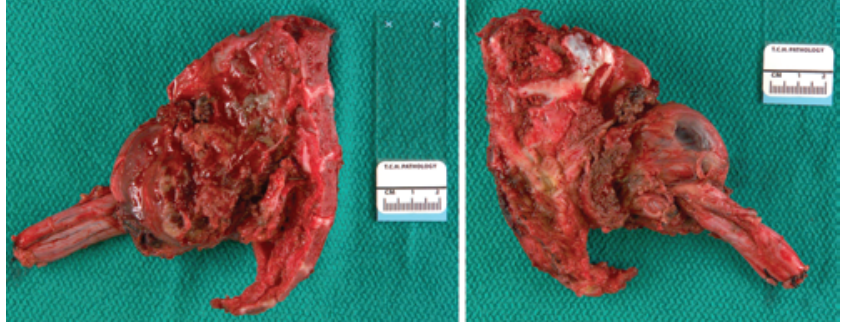

FIG. 2. Gross pathological specimen, dorsal (left) and medial (right) views, obtained after resection of the right hemisacrum, tumor, and sciatic nerve.

truded into the sciatic notch. The distal sciatic nerve was sectioned after determining negative tumor margins along the nerve. The rest of the procedure included L-5 to S-5 laminectomies, a midline osteotomy, and disarticulation of the sacrum from the ilium at the right sacroiliac joint, ligation of the right S-1, S-2, and S-3 dural sleeves with double Weck clips, partial right L5-S1 discectomy, and transection of the right $\mathrm{S} 1-5$ nerve roots. The right hemisacrum, together with the residual tumor and stump of the right sciatic nerve, was removed as a single pathological specimen (Fig. 2).

After bilateral L3-5 pedicle screws and bilateral double iliac screws were placed, 2 transverse 5.5 -mm rods (transiliac bars) were placed, connecting the 2 sets of iliac bolts. Next, longitudinal pedicle screw rods were secured to the rod that was attached to the transiliac rods with lateral and domino connectors. Cross-connectors were placed in 2 locations: between the lumbar rods and between the transiliac bars. Then, a fibular free flap was cut to fit the defect between the ipsilateral ilium and the remaining sacrum. This fibular graft was wired in place utilizing 2 titanium wires (Figs. 3 and 4). Morselized fibular autograft, cancellous allograft bone chips, and demineralized bone matrix were placed after decortication of the L-3, L-4, and L-5 transverse processes and the lateral aspect of the ilia. The previously mobilized rectus abdominis myocutaneous pedicle flap was advanced through the pelvis and was used to reconstruct the sacral defect.

\section{Pathological Analysis}

The specimen revealed a dense cellular, predominantly spindle cell tumor with significant nuclear pleomorphism and nuclear crowding, elevated mitotic activity (up to 5 per $10 \mathrm{hpf}$ ), elevated MIB-1 nuclear labeling index, and multiple foci of coagulative tumor necrosis. These histological and immunohistochemical features are diagnostic of MPNST, or neurofibrosarcoma.

\section{Postoperative Course}

The patient, who had a long in-hospital postsurgical course, responded favorably to intensive physical rehabilitation. Her postoperative course was complicated by the development of a superficial wound dehiscence without signs of infection and the occurrence of an eschar, which needed debridement in the operating room. One month following surgery, she was able to ambulate with the help of a walker and an ankle-foot orthosis on the right side. She exhibited normal strength in her right leg with the ex- 


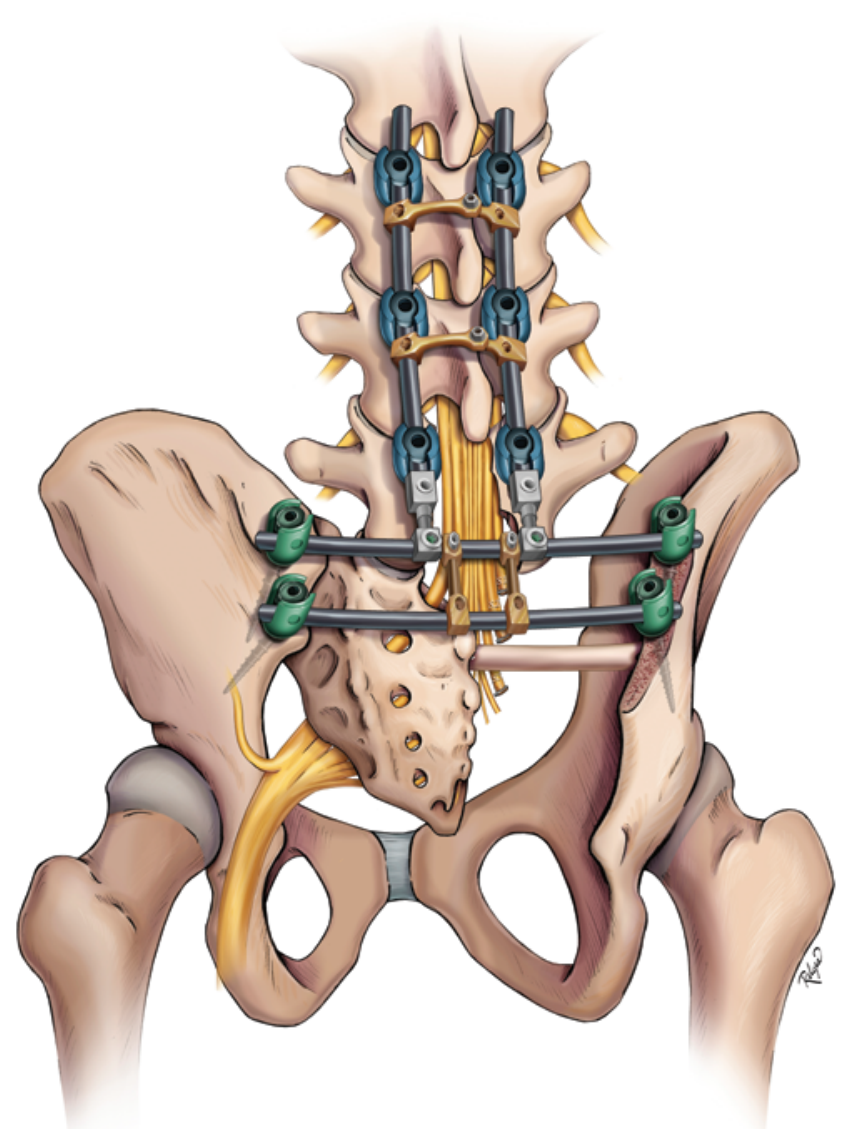

FIG. 3. Artist's illustration depicting lumbopelvic reconstruction after hemisacrectomy for gross-total resection of a sacral tumor. Illustration by Katherine Relyea. Copyright Baylor College of Medicine/Texas Children's Hospital. Published with permission.

ception of plantar flexion (S-1). She was discharged home, completed a course of radiation therapy, and was pain-free off narcotics.

At 25 months after surgery, she remained pain free. She is able to ambulate independently with a right ankle-foot orthosis. She is also able to void spontaneously; however, a voiding cystourethrography demonstrated Grade III vesicoureteral reflux and moderate postvoid residual urine. Therefore, clean intermittent catheterization after each void was recommended. Anorectal manometry and perineal sensation testing revealed normal defecation dynamics with a normal sensation threshold. Subsequently, she had an ileostomy takedown.

Follow-up imaging showed no evidence of residual, recurrent, or metastatic disease. Postoperative radiographs revealed no evidence of any instrumentation failure (Fig. 5).

\section{Discussion}

\section{Neurofibrosarcoma}

Neurofibrosarcoma, or MPNST, is a spindle cell sarcoma that arises from peripheral nerve or demonstrates nerve sheath differentiation. It is a rare disease in children, making up only $4 \%-10 \%$ of all soft-tissue sarcomas in that population. Neurofibrosarcomas are of neuroecto-

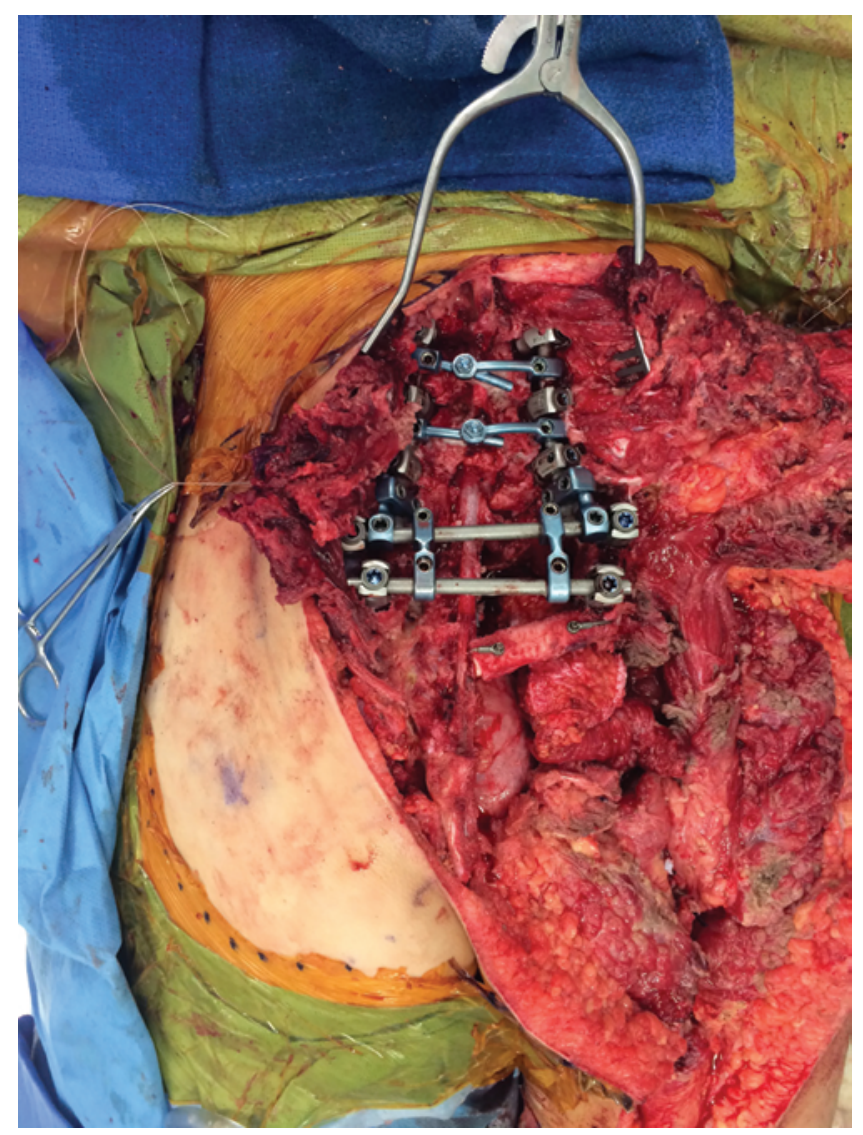

FIG. 4. Intraoperative photograph showing the lumbopelvic reconstruction following hemisacrectomy.

dermal origin, unlike other soft-tissue sarcomas that are of mesenchymal cell origin. ${ }^{19}$ Despite its general rarity, neurofibrosarcoma is one of the most frequent nonrhabdomyosarcomatous sarcomas that occur in the pediatric population. ${ }^{4}$ Therefore, neurofibrosarcoma should be considered in the differential diagnosis of a soft-tissue mass in a child, especially in one with a history of neurofibromatosis or who has previously received radiation therapy. ${ }^{16}$

This tumor has an aggressive nature and a poor natural history with a propensity toward local and distant recurrence, particularly in the lungs. ${ }^{7,20}$ Available clinical data ${ }^{16}$ indicate that the best chance for disease-free survival is complete resection. Gross disease demonstrates little to no response to chemotherapy or radiation therapy. Surgery remains the mainstay of therapy, followed by radiation therapy for microscopic disease in all patients, even if the surgical margins are negative. ${ }^{16}$

Unfortunately, pediatric patients with neurofibrosarcoma have a poor prognosis and a high incidence of relapse. ${ }^{16}$ More aggressive therapeutic regimens are necessary to control both local and distant relapses. Patients are at risk for both of these late recurrences usually 1-2 years after surgery as well as second malignancies. Therefore, surveillance should be lifelong.

\section{Surgical Treatment}

Treating primary sacral tumors presents a challenge given their anatomical location and their usually large 

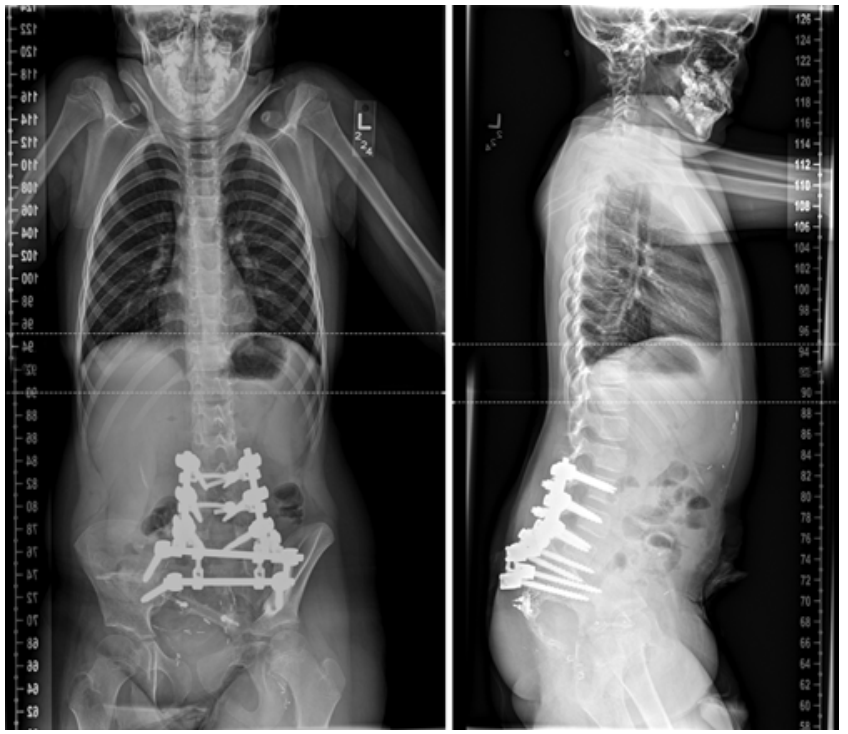

FIG. 5. Postoperative anteroposterior (left) and lateral (right) 36-inch full spine radiographs obtained 2 years after surgery, showing no evidence of instrumentation failure.

size at presentation. Surgical treatment options include partial or total sacrectomy, with or without spinal instrumentation for reconstruction of the lumbopelvic junction. Optimal surgical treatment of these sacral lesions is established by numerous factors, including a patient's preoperative status, the anatomical characteristics specific to the patient and lesion, and the biology of the tumor itself. ${ }^{18}$ Resection often involves interruption of the sacroiliac joint, which links the pelvis and the spine and functions to transmit force during sitting and standing. Therefore, with more than $50 \%$ resection of the sacroiliac joint, instability and consequent axial back pain can result. ${ }^{22}$ Reconstruction of the lumbopelvic junction when sacroiliac joints are invaded by tumor or resected with the surgical margin stabilizes the base of the spinal column with the potential to reduce pain and improve functionality. Patients must be carefully selected as both complete and partial sacrectomies are highly morbid and can result in permanent lower-extremity motor or sensory deficits and loss of bowel, bladder, or sexual function. ${ }^{23}$ This aggressive surgical approach has been shown to prolong the progressionfree survival period in patients harboring various primary sacral neoplasms such as infiltrative benign tumors and malignant tumors that are not responsive to neoadjuvant chemotherapy or radiation therapy. ${ }^{2,3,14,17,21,24-26}$

\section{Hemisacrectomy}

When a resection margin can be defined on preoperative MRI and fine-cut CT scans, and sacral nerve roots on the contralateral side are beyond the area of resection, hemisacrectomy, in which the ipsilateral sacroiliac joint is resected together with the tumor, may be considered.

Postoperative urinary and fecal incontinence could lead to an impaired quality of life for the patient, as well as increased health care costs to the individual and to society. ${ }^{15}$ In hemisacrectomy with the preservation of contralateral sacral nerve roots, a better quality of life can be expected with conserved bladder and bowel function. To confirm this, we compiled a summary of articles reporting the functional outcomes of patients after total hemisacrectomy involving unilateral sacrifice of sacral nerve roots for the treatment of primary tumors of the sacrum (Table 1). In total, 6 articles reported on 31 patients. As regards bladder function, $22(71 \%)$ of the 31 patients had normal bladder function, 6 (19\%) required intermittent catheterization, and $3(10 \%)$ had permanent urinary disorder. In terms of bowel function, $30(97 \%)$ of 31 patients were reported to be normal (that is, did not require a colostomy). These findings are consistent with those in older literature suggesting that unilateral loss of sacral nerve roots does not affect overall bowel and bladder function. ${ }^{10-12}$

Hemisacrectomy can be performed through a 2-stage anterior-posterior approach ${ }^{15}$ or through a single-stage posterior-only approach..$^{5}$ A posterior-only approach has the advantages of decreased surgical morbidity through the avoidance of the anterior approach, shorter operative times, and decreased blood loss. Disadvantages of the posterior-only approach include restricted access to vital structures anterior to the ilium and sacrum, such as the iliac arteries and veins, especially when an emergency arises from injury to these structures. Furthermore, the posterior-only approach limits options available for wound closure. And wound closure is especially important for sacral tumors since the rate of wound infection after the resection of these lesions is high. 5

In children, we recommend a staged anterior-posterior approach that will facilitate identification and protection of the pelvic organs, major blood vessels, and ipsilateral sacral nerves and lumbosacral plexus. A posterior-only approach $^{5}$ is an option in adults; however, its disadvantages and risks outweigh its advantages in children. The anterior-posterior approach allows the specimen to be freed anteriorly from adjacent pelvic organs and major blood vessels before moving onto the definitive posterior stage.

Life-threatening blood loss from injury to the great vessels of the abdomen and pelvis in a child is a major consideration when undertaking the 2-stage anteriorposterior approach. At the time of her surgery, our patient weighed $26 \mathrm{~kg}$ for an approximate effective circulating blood volume of $1.8 \mathrm{~L}$. Even without evidence of arterial and venous injury, our patient lost $1.2 \mathrm{~L}$ of blood from the anterior approach and $1 \mathrm{~L}$ of blood from the posterior approach. Staging the anterior-posterior approach decreases operative and anesthetic time per session, attenuates the physiological insult from surgery and anesthesia to a young child, and allows the patient to be extraoperatively stabilized before proceeding to the next stage.

Furthermore, examination of the ventral surface of the sacrum allows for the planning of a sagittal cut in the sacrum with an adequate margin from the base of the tumor. An anterior-posterior approach also allows mobilization of some structures, such as the vertical rectus abdominus flap, to repair soft-tissue defects following sacrectomy.

The use of a vascularized soft-tissue flap is particularly important in a young child with relatively thin subcutaneous and muscle layers to begin with. Moreover, when a patient assumes a sitting posture, external pressure on the soft tissue overlying prominent lumbopelvic spinal 
TABLE 1. Functional outcomes of patients after total hemisacrectomy

\begin{tabular}{|c|c|c|c|c|}
\hline Authors \& Year & $\begin{array}{c}\text { No. of } \\
\text { Patients }\end{array}$ & Etiology & Bladder Function & $\begin{array}{l}\text { Bowel } \\
\text { Function }\end{array}$ \\
\hline Randall et al., 2005 & 4 & Unspecified (4) & $4 / 4$ normal & $4 / 4$ normal \\
\hline Sabourin et al., 2009 & 5 & $\begin{array}{l}\text { Ewing's recurrence (2), chondrosarcoma (2) Ewing's } \\
\text { sarcoma (1) }\end{array}$ & $3 / 5$ permanent urinary disorder, $2 / 5$ normal & $5 / 5$ normal \\
\hline Wang et al., 2012 & 5 & Osteosarcoma (3), malignant fibrous histiocytoma (2) & $5 / 5$ normal & $5 / 5$ normal \\
\hline Li et al., 2014 & 15 & $\begin{array}{l}\text { Chrondrosarcoma (5), Ewing's sarcoma (3), osteosarcoma } \\
\text { (2), MPNST (2), RCC metastasis (2), malignant GCT (1) }\end{array}$ & 10/15 normal, 5/15 ClC & $15 / 15$ normal \\
\hline Verlaan et al., 2015 & 1 & Chrondrosarcoma & $\mathrm{CIC}$ & Colostomy \\
\hline Clarke et al., 2014 & 1 & Ewing's sarcoma & Normal & Normal \\
\hline
\end{tabular}

$\mathrm{CIC}=$ chronic intermittent catheterization; $\mathrm{GCT}=$ giant cell tumor RCC = renal cell carcinoma.

instrumentation can cause ischemia to the skin and subsequent wound breakdown. In our patient, the additional soft-tissue coverage from a vertical rectus abdominus flap helped to attenuate the prominence of the lumbopelvic spinal instrumentation and to prevent subsequent erosion through the skin.

Our patient had bony anatomy suitable for adult-sized spinal instrumentation. We anticipate that this procedure could be performed in children as young as 2 years of age $^{9}$ with the use of smaller spinal instrumentation, such as those screws and rods designed for the adult cervical spine. Pelvic fixation performed in a skeletally immature child would be expected to cause stunted growth of the pelvic ring.

Should our patient have a normal life expectancy, we would be concerned about her future pregnancies. We previously described our experience with a young girl who had undergone complete sacrectomy and successfully carried 2 pregnancies to term. ${ }^{1}$ We would recommend urinary tract infection (UTI) prophylaxis during pregnancy, as our patient probably has a neurogenic bladder and is at risk for UTIs-and UTIs can precipitate preterm labor. We would also recommend cesarean section for delivery. However, great care must be taken to identify the right inferior epigastric vessels, the pedicle of the rectus abdominis musculocutaneous flap used for closure of the sacral defect.

Unfortunately, other longitudinal studies of patients who have undergone complete or partial sacrectomy are lacking. As mentioned previously, our patient requires lifelong follow-up. We plan to provide periodic updates and insights to the readership regarding the long-term effects of hemisacrectomy at a young age, including biomechanical effects such as adjacent level disease.

\section{Conclusions}

At our institution, we treated a 7-year-old girl with hemisacrectomy with preservation of contralateral sacral nerve roots for gross-total resection of a sacral neurofibrosarcoma. Herein, we describe our technique of lumbopelvic reconstruction. Our goals in the surgical treatment of these primary sacral tumors were to achieve prolonged disease-free survival and to improve overall quality of life and patient functionality, especially bladder and bowel function. The optimal treatment for childhood neurofibrosarcoma is as yet unknown given the rarity of this en- tity. Wide resection with clean margins appears desirable; however, achieving this largely depends on tumor location and local anatomy.

\section{References}

1. Barsan VV, Briceño V, Gandhi M, Jea A: Long-term followup and pregnancy after complete sacrectomy with lumbopelvic reconstruction: case report and literature review. BMC Pregnancy Childbirth 16:1, 2016

2. Bergh P, Gunterberg B, Meis-Kindblom JM, Kindblom LG: Prognostic factors and outcome of pelvic, sacral, and spinal chondrosarcomas: a center-based study of 69 cases. Cancer 91:1201-1212, 2001

3. Bethke KP, Neifeld JP, Lawrence W Jr: Diagnosis and management of sacrococcygeal chordoma. J Surg Oncol 48:232-238, 1991

4. Carli M, Ferrari A, Mattke A, Zanetti I, Casanova M, Bisogno G, et al: Pediatric malignant peripheral nerve sheath tumor: the Italian and German soft tissue sarcoma cooperative group. J Clin Oncol 23:8422-8430, 2005 (Erratum in J Clin Oncol 24:724, 2006)

5. Clarke MJ, Zadnik PL, Groves ML, Dasenbrock HH, Sciubba DM, Hsu W, et al: En bloc hemisacrectomy and internal hemipelvectomy via the posterior approach. J Neurosurg Spine 21:458-467, 2014

6. Doita M, Harada T, Iguchi T, Sumi M, Sha H, Yoshiya S, et al: Total sacrectomy and reconstruction for sacral tumors. Spine (Phila Pa 1976) 28:E296-E301, 2003

7. Doorn PF, Molenaar WM, Buter J, Hoekstra HJ: Malignant peripheral nerve sheath tumors in patients with and without neurofibromatosis. Eur J Surg Oncol 21:78-82, 1995

8. Fourney DR, Rhines LD, Hentschel SJ, Skibber JM, Wolinsky JP, Weber KL, et al: En bloc resection of primary sacral tumors: classification of surgical approaches and outcome. J Neurosurg Spine 3:111-122, 2005

9. Gressot LV, Mata JA, Luerssen TG, Jea A: Surgical treatment of congenital thoracolumbar spondyloptosis in a 2-year-old child with vertebral column resection and posterior-only circumferential reconstruction of the spine column: case report. J Neurosurg Pediatr 15:207-213, 2015

10. Gunterberg B, Kewenter J, Petersén I, Stener B: Anorectal function after major resections of the sacrum with bilateral or unilateral sacrifice of sacral nerves. Br J Surg 63:546554, 1976

11. Gunterberg B, Norlén L, Stener B, Sundin T: Neurourologic evaluation after resection of the sacrum. Invest Urol 13:183188,1975

12. Gunterberg B, Petersén I: Sexual function after major resections of the sacrum with bilateral or unilateral sacrifice of sacral nerves. Fertil Steril 27:1146-1153, 1976 
13. Humphries WE III, Satyan KB, Relyea K, Kim ES, Adesina AM, Chintagumpala M, et al: Low-grade myofibroblastic sarcoma of the sacrum. J Neurosurg Pediatr 6:286-290, 2010

14. Kaiser TE, Pritchard DJ, Unni KK: Clinicopathologic study of sacrococcygeal chordoma. Cancer 53:2574-2578, 1984

15. Li D, Guo W, Tang X, Yang R, Tang S, Qu H, et al: Preservation of the contralateral sacral nerves during hemisacrectomy for sacral malignancies. Eur Spine J 23:1933-1939, 2014

16. Neville H, Corpron C, Blakely ML, Andrassy R: Pediatric neurofibrosarcoma. J Pediatr Surg 38:343-346, 2003

17. Ozaki T, Flege S, Liljenqvist U, Hillmann A, Delling G, Salzer-Kuntschik M, et al: Osteosarcoma of the spine: experience of the Cooperative Osteosarcoma Study Group. Cancer 94:1069-1077, 2002

18. Randall RL, Bruckner J, Lloyd C, Pohlman TH, Conrad EU III: Sacral resection and reconstruction for tumors and tumor-like conditions. Orthopedics 28:307-313, 2005

19. Sabourin M, Biau D, Babinet A, Dumaine V, Tomeno B, Anract $P$ : Surgical management of pelvic primary bone tumors involving the sacroiliac joint. Orthop Traumatol Surg Res 95:284-292, 2009

20. Sciubba DM, Petteys RJ, Garces-Ambrossi GL, Noggle JC, McGirt MJ, Wolinsky JP, et al: Diagnosis and management of sacral tumors. J Neurosurg Spine 10:244-256, 2009

21. Taxy JB, Battifora H, Trujillo Y, Dorfman HD: Electron microscopy in the diagnosis of malignant schwannoma. Cancer 48:1381-1391, 1981

22. Vauthey JN, Woodruff JM, Brennan MF: Extremity malignant peripheral nerve sheath tumors (neurogenic sarcomas): a 10-year experience. Ann Surg Oncol 2:126-131, 1995

23. Verlaan JJ, Kuperus JS, Slooff WB, Hennipman A, Oner FC: Complications, secondary interventions and long term morbidity after en bloc sacrectomy. Eur Spine J 24:2209-2219, 2015
24. Wang J, Tang Q, Xie X, Yin J, Zhao Z, Li Z, et al: Iliosacral resections of pelvic malignant tumors and reconstruction with nonvascular bilateral fibular autografts. Ann Surg Oncol 19:4043-4051, 2012

25. York JE, Kaczaraj A, Abi-Said D, Fuller GN, Skibber JM, Janjan NA, et al: Sacral chordoma: 40-year experience at a major cancer center. Neurosurgery 44:74-80, 1999

26. Zhang HY, Thongtrangan I, Balabhadra RS, Murovic JA, Kim DH: Surgical techniques for total sacrectomy and spinopelvic reconstruction. Neurosurg Focus 15(2):E5, 2003

\section{Disclosures}

The authors report no conflict of interest concerning the materials or methods used in this study or the findings specified in this paper.

\section{Author Contributions}

Conception and design: Jea. Acquisition of data: Palejwala, Fridley, Garcia. Analysis and interpretation of data: Palejwala, Fridley, Garcia, Vasudevan, Khechoyan, Rednam, Koh. Drafting the article: Jea, Palejwala. Critically revising the article: Jea. Reviewed submitted version of manuscript: Jea. Approved the final version of the manuscript on behalf of all authors: Jea. Administrative/technical/material support: Vasudevan, Khechoyan, Rednam, Koh. Study supervision: Jea.

\section{Correspondence}

Andrew Jea, Indiana University School of Medicine/Riley Hospital for Children, 705 Riley Hospital Dr., Indianapolis, IN 46202. email: ajea@goodmancampbell.com. 